\section{Risques et sécurité de l'utilisation des médicaments à l'hôpital}

P. Muff ${ }^{a}$, R. Egger ${ }^{b}$, M. Lysser ${ }^{c}$, P. Meier-Abt ${ }^{d}$, J.-P. Reymond ${ }^{e}$, D. Scheidegger ${ }^{f}$

a Dr, pharmacien hospitalier, Hôpital cantonal Fribourg; président du congrès

b Dr, pharmacien hospitalier, Hôpital de Langenthal

c Responsable de l'informatique pour les soins infirmiers, Hôpital universitaire de Zurich

d Prof., pharmacologie clinique et toxicologie, Hôpital universitaire de Zurich

PD Dr, pharmacien hospitalier, ICHV, Sion

f Prof., département d'anesthésie, Hôpital cantonal de Bâle

Du 24 au 26 avril 2001 a eu lieu à Fribourg le premier congrès national interdisciplinaire sur le thème "Risques et sécurité: le médicament à l'hôpital", auquel ont participé plus de 250 personnes. Organisé par des infirmiers, médecins et pharmaciens des hôpitaux, ce congrès avait pour but de faire le point sur la problématique du risque médicamenteux hospitalier selon une approche systémique. Les exposés de personnes directement concernées (les patients, les soignants et les médecins) ont été suivis par ceux de spécialistes qui ont analysé l'ensemble des facteurs pouvant influencer les prestations des personnes impliquées dans le circuit du médicament. De nombreuses idées et solutions prometteuses pour gérer ces problèmes ont été proposées et discutées. Pour soutenir ce changement d'attitude et de vision qui implique l'intégration de la notion du risque dans la pratique professionnelle, les médecins, soignants et pharmaciens ont décidé de créer une base de données commune permettant de saisir les incidents médicamenteux.

\section{Les complications médicamenteuses sont fréquentes ...}

Bien qu'en soins aigus, le médicament soit un des moyens thérapeutiques le plus important, de nombreuses études ont démontré qu'ils sont également

Correspondance:

PD Dr pharm. Jean-Philippe Reymond

ICHV

Division de Pharmacie

Grand-Champsec 89

Case postale 736

CH-1951 Sion

E-mail: jphilippe.reymond@ichv.vsnet.ch une des causes les plus fréquentes de complications: en fait, plus de $10 \%$ des patients hospitalisés sont concernés par une complication d'origine médicamenteuse. Elles sont pour la plupart banales mais parfois graves voire mortelles et peuvent générer des surcoûts énormes pour la santé publique. Environ un tiers des complications médicamenteuses est imputable à des erreurs qui auraient pu être évitées.

En avril 2000, les sociétés suisses des pharmaciens de l'administration et des hôpitaux GSASA, des médecins-chefs des hôpitaux VLSS et des soignants ASI ont par conséquent décidé de débattre en public du thème des risques inhérents à l'utilisation de médicaments en milieu hospitalier.

A l'époque, il était peu question d'erreurs en santé publique et il paraissait utopique de croire que trois groupes professionnels si différents puissent se réunir un jour dans le but de rechercher ensemble des solutions sur la seule et simple prise de conscience du problème. Toutefois, du 24 au 26 avril plus de 250 participants sont venus au Forum Fribourg.

\section{... et soulèvent des questions fondamentales}

Le premier but du congrès était de faire le point sur la problématique du risque médicamenteux hospitalier. Puis il s'agissait de mettre en évidence les connexions et relations dans le système de santé afin de comprendre et d'améliorer durablement la sécurité d'utilisation du médicament à l'hôpital, et finalement de convenir de mesures concrètes.

C'est d'abord le point de vue de la "première victime" (le patient) qui a été présenté suivi de celui des "secondes victimes" (les soignants et les médecins prescripteurs). Un spécialiste des industries à haut risque a ensuite expliqué qu'il fallait apprendre à passer d'une perspective individuelle à une approche systémique pour assurer la sécurité en général et celle de l'emploi des médicaments en particulier. Des spécialistes de différents domaines ont ensuite présenté les facteurs qui influencent les prestations des personnes impliquées dans le circuit du médicament. En effet, la sécurité d'utilisation des médicaments à l'hôpital ne dépend pas uniquement des compétences des utilisateurs mais également des caractéristiques des produits et de facteurs externes tels que l'ergonomie de la place de travail, le fonctionnement de l'équipe, l'organisation de l'hôpital ainsi que des contextes légaux, économiques, sociaux et politiques.

\section{Des problèmes systémiques requièrent des solutions systémiques}

De nombreuses idées et solutions prometteuses pour gérer ces problèmes ont été présentées et discutées: amélioration de l'identification des produits et de leur mode d'emploi, apprentissage et perfectionnement de la compétence à travailler en équipe, mise en place d'un climat de confiance dans l'hôpital, saisie des in- 
cidents médicamenteux, distribution des médicaments sous forme de doses unitaires, préparation centralisée de médicaments iv dangereux, prescription informatisée, implication d'un pharmacien clinicien, etc.

Or, il est apparu que la plupart des solutions évoquées ne peuvent être réalisées que si elles bénéficient du soutien des autorités des politiciens et des médias. Les nouvelles mesures de sécurité pourraient être alors financées grâce à un transfert des moyens financiers utilisés actuellement pour traiter les complications.

\section{Changement de vision, aller vers une culture du risque!}

Les objectifs du congrès tels que la sensibilisation et le renforcement de la collaboration entre les trois groupes professionnelles ont été atteints avant même la fin du congrès. Comme première mesure concrète, la FMH, l'ASI et la GSASA ont décidé d'adapter la base de données développée par le Département
d'Anesthésie de l'hôpital cantonal de Bâle (CIRS) de telle manière à ce que les trois groupes professionnels puissent l'utiliser pour saisir de façon aussi étendue que possible et au niveau national les incidents médicamenteux. Cette approche doit soutenir le changement de vision qui implique d'intégrer dans son travail quotidien la notion de culture du risque et de contribuer à faire reconnaître, en dehors de l'hôpital aussi, les problèmes qui peuvent se produire dans le circuit du médicament.

Le nombre important de participants a également démontré que dans toutes les professions impliquées il existe de nombreuses personnes qui ont reconnu l'importance de ce problème et qui veulent s'engager à faire appliquer ces changements.

Grand merci à toutes celles et à tous ceux qui ont contribué au succès de ce $1^{\text {er }}$ congrès interdisciplinaire sur la sécurité du médicament à l'hôpital.

Les personnes intéressées trouvent l'abstractbook des présentations du congrès sous http://www.gsasa.ch/ gsasa/index.html

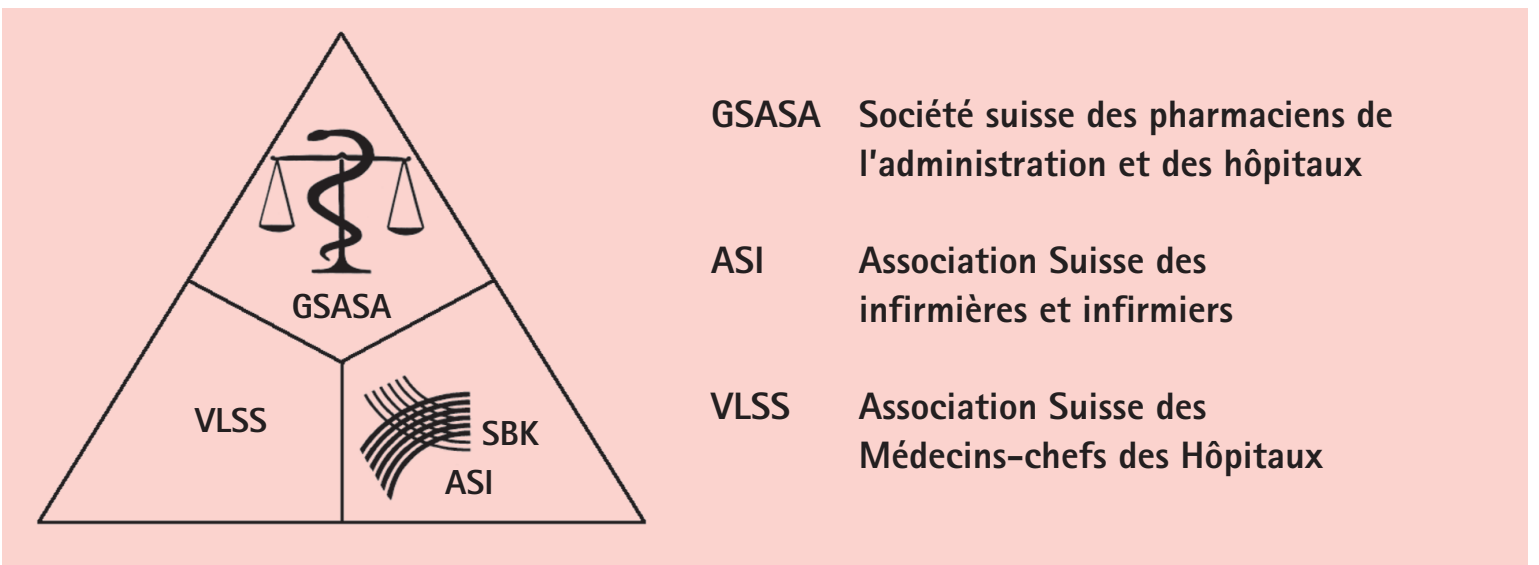

